\title{
Experience and Vision of Open Innovations in Russia and Baltic Region: the FRUCT Program
}

\author{
Sergey Balandin \\ Nokia Research Center, Helsinki, Finland, Sergey.Balandin@nokia.com
}

\begin{abstract}
This paper discusses our vision and experience of developing Open Innovations framework program, how it was implemented and why. The Open Innovations paradigm is a new trend for performing research and development that was proposed only a few years ago in 2003. The main emphasis of this approach is on setting cooperation in form of direct industry-to-academia joint $R \& D$ project and development of the corresponding competence incubators around the most relevant technologies. As an example this paper presents the open innovations framework program FRUCT that is targeted in development of telecommunications R\&D ecosystem in Russia and Baltic region. The need for such cooperation is already recognized by the local industry, academic community and government authorities. In particular, Nokia and Nokia Siemens Networks expressed readiness and interest to invest into this project by contributing competences and providing some financial support.
\end{abstract}

Keywords: Open Innovations; FRUCT; Industry-toAcademia; $R \& D$ cooperation; new trends in innovations; Russia-Finland partnering; Russia-Baltic cooperation.

\section{INTRODUCTION}

The paper presents a project targeted in developing mobile $R \& D$ ecosystem of Russia and Baltic region, with the main emphasis on setting up industry-to-academia competence incubator operating in the format of open innovations. Continues development of the strategic partnership between industrial and academic research is a key success factor of the modern innovation ecosystem. There are a few success stories of such strategic partnership frameworks functioning in different parts of the world. These programs bring significant benefits to the involved parties and fueling their further R\&D units. As we know the fundamental science driven by the universities and other academic organizations should not be directly attached to the existing industries, but industrial research would benefit by early access to the results and information about main trends and weak signals. At the same time many universities also active in the applied research, but to be efficient they need feedback channel from the industry. Another key driver for setting stronger connection between academia and industry is that the time between a moment of innovation and its adoption by the industry is getting shorter and shorter. An interesting new trend for addressing this need is by building open innovation frameworks targeted in developing strategic partnership between industrial and academic research. Such framework programs help to find right research partners and jointly incubate new competences.
Nowadays the USA universities are the recognized leaders in adaptation of the academic research and education to the existing industrial needs, just look to the density of industrial presence in Silicon Valley. This situation creates a strong demand for quick and adequate actions from universities in Russia and Europe. A number of cooperation frameworks have been built inside the EU, e.g., Framework Program 7 [1]. However, the cooperation between Europe and Russia is still leaves a lot to be desired. This creates a historical chance for Finnish universities to use geographical proximity and traditionally good relations with Russian colleagues to strengthen Finnish science. Such cooperation is in clear mutual benefit, as among other advantages it will give to Finnish academia a priority path for accessing the huge pool of highly qualified talents and new innovative competences and help Russian universities to better integrate into the EU academic institutions and consequently will contribute in development of the bridge between academic and R\&D worlds of EU and Russia.

There is a number of well known and hidden thresholds on the road to long-term collaboration and partnering. The most critical are need of mutual trust, lack of awareness about partners' capabilities, need for significant initial investments, and so on. As a result many good collaboration opportunities die at the very initial phase. This is especially true when thinking of $R \& D$ cooperation in countries that do not have long history of cooperation with global industry players. At the same time these regions have large undiscovered R\&D potential, e.g., "nontraditional" solutions, new bright ideas that are not well known outside of a particular team and so on. Industrial research can benefit by getting early access to this "box of secrets" and the first players that manage to achieve it will win the most.

This paper describes our experience of building, managing and developing Finnish-Russian University Cooperation in Telecommunications (FRUCT) Open Innovations Framework program [2]. The FRUCT program was established in 2007 by a group of enthusiasts supported by Nokia and two universities. By now the program unites teams from 18 universities, Russian Academy of Science and is supported by Nokia and Nokia Siemens Networks, the companies that are recognized long-standing leaders and drivers in their segments of the ICT industry.

In particular, the paper focuses on two key aspects of the FRUCT program, which are less understood by the 
outside observers, but are the key factors contributing to the success of program:

- Main elements and principles of FRUCT framework as a whole and our approach to the program management and technological steering;

- Principles of organizing, managing and sharing results of the joint R\&D project between FRUCT member teams.

The experience of presenting FRUCT principles to externals and followers shows that the solutions for these two points are the most complicated for understanding of FRUCT and Open Innovation paradigm. So this was an original motivation to prepare the paper and put these topics to the open discussion in R\&D community.

The paper is organized as follows. The next section gives an overview of FRUCT mission, motivation for the member organizations, general principles of operation, expected deliverables and achievements. The third section specifically addresses core principles of FRUCT management on the level of whole program and each particular project. The main points of this paper are summarized in conclusion section, which is followed by acknowledgements and the list of references used in the study.

\section{GENERAL OVERVIEW OF THE FRUCT PROGRAM}

This chapter gives an overview of FRUCT program, its mission and main principles of operation. The theoretical basement of the program is the principle of open innovations proposed by Prof. Henry Chesbrough from UC Berkley [3]. Open Innovations is a paradigm that assumes that firms can and should use external ideas as well as internal ideas, and internal and external paths to market, as the firms look to advance their technology [4]. The boundaries between a firm and its environment have become more permeable; innovations can easily transfer inward and outward. The central idea behind open innovations is that in a world of widely distributed knowledge, companies cannot afford to rely entirely on their own research, but should instead buy or license processes or inventions from other companies. In addition, internal inventions not being used in firm's business can still give benefits outside the company, e.g., through licensing, joint ventures, spinoffs [5]. However, implementation of the open innovations principle requires significant ecosystem preparation work and framework that provides trust and other components that fuel open innovations.

Nowadays we can claim that the FRUCT program implements main principles of open innovations plus we done further theoretical and practical development of the basic principles in the part related to the ecosystem preparation to open innovations. The program aims in increasing level of competences and visibility of the member organizations, especially awareness about Russian research in Europe and wise versa. Russian universities have good reputation and traditions in fundamental science. However, visibility and presence of young Russian scientist in the international scientific community is a clear area for further improvement. Historically the first area of the corresponding development selected by FRUCT was development of competences and infrastructure that allows young talents to participate and publishing papers at the top international conferences. At the same time European, e.g., Finnish universities gets this way the great opportunity to access the largest pool of talents and resources in Europe.

The FRUCT program was established in 2007 by the group of individuals, Nokia Research Center, SaintPetersburg University of Airspace Instrumentation and University of Turku. Originally the program was targeted in facilitating cross-boarder $\mathrm{R} \& \mathrm{D}$ cooperation between industrial and academic organizations of Finland and Russia. By now FRUCT become the most significant and actively growing cooperation framework between leaders of ICT industry and universities in the Baltic region. At the moment the FRUCT community consists of representatives of 18 universities from Russia, Finland and Denmark, three industrial companies represented by their R\&D units, and R\&D institute of Russian Academy of Science. The main FRUCT principle is in removing cooperation entering thresholds by setting $R \& D$ projects run by students under direct joint supervision and tutoring of industrial and academic experts. The FRUCT principle of building cooperation through the joint student-driven R\&D projects has been proven to be very efficient for identifying and incubating the demanded competencies. The big advantage is that initially all cooperating sides take minimal obligations, with low financial and image risks. As a result a number of cooperation activities have been started and successfully developing. Care of the personal development of involved people and teams is the key priority of the program. The FRUCT students have successful represented the program in a series of international contests, including Symbian Student Essay contests, Widsets coding contests, and so on. Also a number of good international publications have been done within the FRUCT scope and we scale of activities is growing fast.

Generally FRUCT program promotes mobile device oriented research, telecommunication and information technologies. The directions of research within the FRUCT program include (but are not limited to) open source solutions and MeeGo/MAEMO mobile OS, smart spaces, physical air interface, embedded networks, mobile device software and service solutions, energy management and green technologies, security, and so on.

FRUCT program is based on two modes of cooperation: cooperation of R\&D team in joint projects and regular face-to-face meeting (i.e., conferences, seminars, trainings, etc.) for gathering together all members of the FRUCT community. The R\&D projects cooperation helps to the involved teams to learn about the capabilities of each other, building thus basic trust and understanding. The fact that projects are done by students is important as it minimize the involved costs and risks. Our approach focused on creating international groups of students supervised by industrial and university experts, which help directing the R\&D work of the students in the most interesting and challenging areas of ICT R\&D. In other words, 
the program implements the project-based training, where students are oriented towards real creativity and contributing to the final concrete deliverables. Generally it is hard for industry and academy people to find the right partner for cooperation, but such small joint R\&D projects provide the required basic interface and topic for setting direct contacts and find ground for commercial projects.

The key enabler factor of success in development of the strategic cooperation is to identify solid and well established niche where partners have unique and supplementing competences. The FRUCT program creates an environment to highlight the existing relevant $R \& D$ niches and what even creates new $R \& D$ niches around recently emerging technologies, which can be generated as a product of open innovation cooperation between industrial and university experts. The FRUCT also helps universities to incubate new competences demand for which is emerging on the industry side. The main goals of FRUCT program include:

- Identifying world-class R\&D teams that are looking for partners and interested in open innovation cooperation;

- Creating the new competences and corresponding niches for R\&D cooperation;

- Developing long-term strategic partnership between industry and universities;

- Providing chance for more students to realize scientific, R\&D and career ambitions at the university through direct academia-to-industry cooperation;

- $\quad$ Promoting idea of Europe without borders and corporate social responsibility.

It is also well known problem that universities experience difficulties in keeping the best students, but close partnering with industry provides association with the strong brands, challenging and very concrete research tasks and additional resources, which attract students and help to solve the resource problem. On the other hand, the industry companies are interested to have long-term and high-risk research done by the universities, and benefit from getting closer to the edge of science, so that adoption of new key finding could be done even faster. Also the early industrial feedback is in mutual benefit as it allows right tuning and presentation of the new technologies. So driving into a stronger cooperation between the academic and industrial research, being more open and involved in joint activities, getting stronger visibility by making joint publications and so on, these all are also in the strong mutual benefit.

One of the most interesting questions is how this rather informal structure exists, how to define and control vector of development, what are the main management principles of FRUCT program.

\section{MANAGEMENT OF THE FRUCT PROGRAM}

The chapter presents two key aspects of FRUCT framework management solution: management of
FRUCT program and management of R\&D projects inside FRUCT. When looking from the program deliverables point of view, these two areas looks like one big task, but in fact in order to achieve efficient management and build robust and scalable solution we shall clearly separate these areas. The main focus of framework management is on maintaining and developing efficient and cozy environment that attracts best talents and creates motivation for people to actively contribute within scope of the open innovations principle. For that the framework must guaranty reliable and equal access to a set of benefits on both individual and group levels. The short summary of the FRUCT member benefits is as follows:

- access to new competences and professional growth opportunities for the involved individuals and teams;

- $\quad$ professional network, trust building and direct contacts with relevant top experts from academia and industry;

- contribution to the positive business and scientific visibility of the member teams and individuals.

Cooperation in the joint projects is the crystallization point that allows providing the above listed benefits, but in order to make it work a proper infrastructure and resource allocation has to be provided. The main FRUCT engine is a group of highly motivated enthusiasts of the open innovations paradigm, who believes that this kind of framework in future will result in incubation of innovative and highly-competitive businesses. Also FRUCT receives some financial support from the industrial members that are interested to develop the overall ecosystem of the region and also care of having image of the good corporate citizen. Together these two factors provides strong enough basis for delivery of the above stated benefits to the program members. It is important to mention that for academic members the program is free of charge and even industrial members have no direct financial obligation, but by default certain level of support is expected. FRUCT makes a lot of investment into development of the efficient environment to support the open innovation principles so that all FRUCT members can for free enjoy the following open innovations project enablers:

- FRUCT conferences (200+ attendees) are organized every half a year, plus FRUCT seminars, topical conferences, workshops and sessions are regularly organized as independent events or within scope of other well respected events in the field of communications;

- technological trainings, exchange lectures and courses, winter and summer schools, open repositories with various materials;

- support of the active FRUCT teams with the research enabling things, such as books, dif- 
ferent kinds of devices, measurement tools, software licenses, project management tools, office equipment, and so on;

- travel grants and free registration/accommodation packages for presenting FRUCT project papers at the recognized conferences and exhibitions;

- $\quad$ R\&D contests with good prizes and opportunity to gain high recognition and good publicity of the project results;

- $\quad$ R\&D grants for students and in certain cases special student stipendiums.

In addition the members get a lot of other supporting benefits that keeps them attached to the program, e.g., discounts on different industrial, academic and scientific events, FRUCT facilitates $\mathrm{PhD}$ and MSc exchange between member universities, help students to find good academic and industrial consultants, team of co-authors for publications and thesis opponents, help forming consortia for participation in EU and national grants, etc. So let's now discuss in details how does it work.

\section{A. Management of the FRUCT framework}

Let's first define what is the FRUCT framework nowadays. FRUCT unites R\&D teams from 22 organizations, located in 3 countries and 4 time zones, with at least 4 major cultural backgrounds. The number of people actively working in FRUCT exceeds 70 persons plus over 200 active followers. Five FRUCT laboratories are located at four Russian universities, in three different cities. The core FRUCT management team is 8 persons. The program is active for more than 3 years and over this time 12 projects were successfully completed and went to further collaboration phase. Over 70 publications were produced as a result of FRUCT activities and over 40 projects currently are under development. FRUCT is leading R\&D activities of Russian MAEMO community and represents interests of Symbian Foundation community in Russia. Internet visibility is supported by the group of FRUCT sites, which includes the main web site, FRUCT forum, five fully operational sites of the second level and three more sites of the second level are under development. Only the main FRUCT site recently gets over 700 views per day. FRUCT follows EU and local calls for project grants, identify the most relevant topics and facilitates formation of the consortia. Also FRUCT carefully follows all new trends and weak signals in ICT industry, which is used for steering technological development of the community. At the same time FRUCT is quite unofficial organization that follows principles of the new Internet era, e.g., it does not have permanent staff $100 \%$ allocated for FRUCT bureaucracy, as all management positions are occupied by people working in the member organizations and having other duties beside the FRUCT activity. At the same time the core management team has significant work load in FRUCT, which create a number of personal and organizational challenges. This is the FRUCT scope. As you can see it is quite broad, challenging and non-trivial solution, so how it is managed?

Formally FRUCT framework is built on the principle of eManagement in matrix organizational structure. The key tools that support FRUCT management are: the existing group of web site and other Internet tools, well defined structure of on-site representation in the member organizations and procedure for internal communications, and regular face-to-face meetings, including social events plus joint free-time activities of FRUCT activists.

The regional representation of FRUCT is based on a network of FRUCT laboratories and teams that are created in the most active partner organizations. The decision to setup FRUCT laboratory should be seen primary as recognition of the existence of strong local FRUCT team and its ability to identify R\&D niche with competitive level of expertise. The lab creation starts from selection of the future lab leader. There are two ways how the lab leader and correspondingly the lab organization can be formed. The lab leader can be recommended and supported by the official authorities of the member organization. Then FRUCT can either accept lab with the proposed leader or refuse it, or start negotiation about other potential candidates. The lab structure in this case is formed by the member organization according inline with the internal guidelines and traditions.

Another approach is used when initiative comes from the department level. Then the laboratory leader is selected via the "natural competition" when at the beginning all key members of the partner team get equal level of recognition and attention from the FRUCT board. This helps to identify natural leader of the team. If the team does not have natural leader, then such case is put on ice till the moment when the leader will appear. If team has more than one natural leader this approach helps us to identify the strongest leader. In certain cases FRUCT might even have two independent FRUCT laboratories in the same university.

Selection of the right person to the laboratory leader position is a key task for any organization, which is absolutely viable requirement for the open innovations type of organization, which by definition are connected by the weak ties and have small and weak bureaucracy structure that definitely cannot handle additional management overhead created due to lack of initiative at the laboratory level.

FRUCT labs get certain amount of money as a budget for internal needs. The money can be used for general expenses of the lab (e.g., traveling, small office expenses, etc.), for organizing lab-level events and support exchange activities, and so on. This gives to the lab and university people some level of financial independency, so that it internally can decide who deserve additional recognition, what additional equipment they need to buy, etc. The lab leaders regularly report the status and devel- 
opment to the FRUCT board and budget stakeholders. The lab leaders together with the FRUCT general chair (and optionally other core team members assigned by the chair) define the lab development plans with horizon of half a year and two years. These plans are reviewed, reevaluated and updated every half a year. Also every half a year the general chair organizes personal development discussion with the leader of each team, which is often held at the time of the main FRUCT conferences. The purpose of this discussion is to understand personal development priorities of the lab leader and team members. The lab lead is responsible for organizing similar discussions with all members of the team. As a result FRUCT cares about interests of the involved people, gets efficient mechanism for getting feedback and minimize probability of unwanted surprises due to loss of people that do not see for themselves benefits in maintaining FRUCT membership in their future. The lab leaders form a sub-team of local leaders; it is recommended that one person is responsible for each area of the strategic development of the laboratory. The package leaders are responsible for running package management tasks, defining and maintaining the work breakdown structures for the projects in their packages and be the main interface for the people involved in the package project work.

Another important aspect is how to handle crosscultural management, defining common language and communication principles. We selected English to be the official language of FRUCT, even despite dominance of Russian participants. However to once again lower the entering barrier, recently FRUCT introduced couple of satellite resources (e.g., maemo.fruct.org) in Russian. But all official email exchange is in English. English is used on all main pages of FRUCT program and in the official news line.

Synchronization of the framework activities is achieved by setting regular telcos in Skype for the members of the core team. In addition, every day the general chair reserves open timeslot for telcos that is convenient for participants in all time-zones. This time is reserved specially for ad-hoc telcos so that any FRUCT member can call to the chair and provide direct feedback, ideas and recommendations. Also every month FRUCT publishes newsletters and sends major announcements via the community email distribution.

The above listed tasks are facilitated by use of Web 2.0 solutions at www.fruct.org, e.g., professional social network engine, blogs, event pages, project pages, lab pages, forum, wiki, inline commenting of news, etc.

The risk management is handled at both framework and project level. At the project level FRUCT use standard risk management planning, plus the lab risk management plan and budget provide the second line of defense against major risks. The third line is provided at the framework level, when all teams operating in cooperation project should have a plan what to do if peer's deliverable will not be available in time.

\section{B. Management of $R \& D$ projects in FRUCT}

As it was said in introduction, the main task of all FRUCT projects is creation of new competences and cooperation contacts between the involved parties. The actual R\&D challenge should be mainly seen as the crystallization point around which the competences and cooperation activity is built.

According to FRUCT rules any representative of the full-member organization can propose a new topic for R\&D project. Every FRUCT project should involve representatives from two or more member organizations. In the simplest case the project work group consists of students from one university, which are supervised by local professor and assigned industrial tutor. In more advanced cases the team might consist of the members from a number of FRUCT universities, industrial experts can directly participate in the research work and more than one supervisor and/or tutor from different universities and companies can assist to the team.

The key idea for such organization of the project teams is to lower the commitment threshold on the way to start real cooperation. It is obvious that before entering into a serious cooperation it would be comfortable for the involved parties to get know each other without any commitments. FRUCT projects provide such "sandbox" framework and additional time to learn about strong and weak sides of the partner. It also helps to get the required knowledge for fine tuning of the cooperation proposals. As a result the proposal can be presented in the format and under conditions that are most favorable for setting the full-scale long-term cooperation.

Another idea behind FRUCT projects is to promote project-based training to help young specialist be more creative and understand how to balance high-risk research with a need to deliver results within the agreed timeline. The eventual results of the project are not just classical deliverables like novel algorithms, signal structures, architectural solutions, software code, etc., but creation of the "competence incubation" infrastructure and a set of well-prepared teams capable of continuing challenging research and design work on their own.

The new FRUCT projects can be initiated by a professor, industry expert or even by a student. Of course, depending on the initiating party there are some differences in the procedure: when a professor initiates project, he/she has to take an obligation to be a supervisor of the project team, when the project proposal comes from the industry expert, the expert takes obligation to be team's tutor. In both these cases FRUCT performs lite-analysis of the project proposal, as the main responsibility for the quality of project proposal is on the initiating expert. When a student comes with the idea of a new project he/she should ask for a supervisor and tutor from the 
FRUCT board. Such project proposals are evaluated by FRUCT Advisory Board members and invited experts. If the selected domain and problem definition look reasonable and challenging, the proposal is called accepted and FRUCT board helps student to find supervisor and tutor.

The main expected deliverables of FRUCT projects are: article in a good journal or/and conference, and/or code contribution to one of the open source initiatives. However depending on the project scope some other deliverables could be also possible.

As was discussed in the previous section, FRUCT does not provide direct financial support of the projects, but all projects can get support in expertise, books and device donations, tools for developing and managing projects, travel grants and free-participation packages for presenting project results at the approved conferences (with reasonable quality and visibility) and in certain cases even student stipendiums. The project results belong to the developer team, so FRUCT does not pretend to the results ownership, only to the parts the FRUCT leadership team contributed to.

In order to be accepted in FRUCT the project must pass standard project definition and evaluation procedure. Taking into account specifics of FRUCT principles and targets we developed own procedure for entering to FRUCT and special form that must be filled in by all candidate projects. The form and procedure steps are available at FRUCT web [1] and in fact represent the first tool that we give to the news teams to correctly and efficiently formulate the project proposal.

Each project must openly report main results and overall progress comparing it to the original targets every half a year at the FRUCT conferences. Plus the status progress reports are regularly sent to the corresponding lab and/or team leaders.

The project steering is performed by the FRUCT advisory board under personal control and responsibility of the selected tutor. For controlling project progress we recommend to the project teams prepare the timeline plan using Gantt chart [6], with clear specification of the main project phases and dates, names of responsible persons, summary of involved resources, plus also reflect major milestones in the status section of the project web page. In addition we introduced web-based Concurrent Versions System (CVS) solution as a part of the new FRUCT project management framework that is currently in pilot use. The web solution is also combined with the commenting, brainstorming, project and individual calendar tool and ideas aquarium tools.

The above listed tasks are facilitated by use of Web 2.0 solutions provided at fruct.org and the laboratory sites: project pages, professional social network engine, event pages, forum, wiki, CVS, calendar, ideas aquarium, brainstorming, feedback tool, whiteboard, etc.

\section{CONCLUSIONS}

The paper gives an overview of the FRUCT framework, its scope and targets, with special attention to the approach for addressing the framework and project management tasks and what tools and solutions are used for that. As FRUCT was one of the first attempts to implement the open innovation principles in Baltic region. In this project we have faced a lot of challenges and had to implement a lot of program components without having reference examples for adoption of the best practices. Development of the proper management framework was the key element of the performed investigation and this study is not finished. FRUCT has working solution in hands, but still a number of questions are open for further study and discussion (e.g., degree of the solution scalability). The described management framework is under development and even some practices and solutions mentioned in the paper are still in piloting and not yet applied not to the whole FRUCT framework and all projects, but only to the selected subset. FRUCT is a "living" organizational structure and we all time try new things and approaches. Unfortunately due to the space restriction it was not possible to describe many other aspects of FRUCT program. Many management activities, such as management of the professional developer communities (maemo.fruct.org), organization of scientific and educational events (conferences, training, courses, $\mathrm{PhD}$ and $\mathrm{MSc}$ exchange, etc.), formation of consortia for applying to public funding and many other aspects were just mentioned in the paper. We believe that the paper provides strong enough arguments in favor of the open innovations paradigm, which are further supported by the reference to successful implementation.

\section{ACKNOWLEDGMENT}

Author thanks all active members of the open innovation framework program FRUCT, and especially Alexey Dudkov, Veronika Prokhorova and members of the FRUCT Advisory Board for their contribution to the overall success of the program. The special thanks also go to Nokia and Nokia Siemens Networks for donations provided to FRUCT program and all FRUCT experts who contribute their time and expertise to support development of open innovation principle in Russia and Baltic region.

\section{REFERENCES}

[1] Official web site of European Commission. Research: Framework Program 7: the future of European Union research policy, 2010. http://ec.europa.eu/research/fp7. Retrieved: 16.7.2010.

[2] Official web site of Finnish-Russian University Cooperation in Telecommunications (FRUCT) Open Innovation Framework program, 2010. http://www.fruct.org. Retrieved: 16.7.2010.

[3] H.W. Chesbrough, Open Innovation: The new imperative for creating and profiting from technology. Boston: Harvard Business School Press, 2003.

[4] H.W. Chesbrough, Open Innovation: The new imperative for creating and profiting from technology. Boston: Harvard Business School Press, p. xxiv, 2003.

[5] H.W. Chesbrough, The era of open innovation. MIT Sloan Management Review, Vol. 44 (3), pp. 35-41, 2003.

[6] KIDASA software. Gantt Charts, http://www.ganttchart.com. Retrieved: 16.7.2010. 